\title{
Data and Knowledge Management in Designing Healthcare Information Systems
}

\author{
Nadeem Mahmood, Aqil Burney, Zain Abbas \\ Department of Computer Science, \\ University of Karachi, Karachi, Pakistan
}

\author{
Kashif Rizwan \\ Department of Computer Science \& IT, \\ Federal Urdu University, Karachi, Pakistan
}

\begin{abstract}
Revolution in information and communication technologies has transformed the information management structure in almost all the organizations. It has had a great impact on healthcare organizations by improving health services and management by integrating technology with the knowledge management infrastructure. The healthcare industry is a knowledge-based community and is connected to hospitals, physicians, patients, laboratories, pharmaceuticals, clinics, pharmacies, and customers for sharing knowledge. A knowledge-based healthcare industry can improve the quality of care and service given to its people and also reduces the administrative cost. The objective of this research is to present and describe the knowledge management capabilities, the technical infrastructure, and the decision support architecture for such a healthcare management system. It envisions a healthcare knowledge management system (HKMS) that would help to integrate important components, disseminate knowledge to the respective users and to store historical data in a database. This will immensely help the managers and developers to identify their IT needs and to plan for and develop the technical infrastructure of the health care management system for their organizations.
\end{abstract}

\section{Keywords}

Knowledge Management, Healthcare, Information System, Decision Support System, Health Management, health ontology, telemedication.

\section{INTRODUCTION}

There is an increasing awareness among health care organizations for building and using Health Information System (HIS) to provide better services and care to patients in a hospital environment [1] [2]. HIS is linked with the clinical decision support system which provides decision support to all levels of healthcare service providers, including doctors, staff and administration. This helps them in taking quick and appropriate decisions. These decisions may range from billing, diagnosis, lab testing, assignments, data analysis etc. as per the needs of an organization [3] [4] [5] [6] [7].

The main objective of this research is to identify and propose a framework for the health care knowledge management system. The proposed framework describes the important functionalities of different components of the $\mathrm{KB}$ and to integrate with ICT infrastructure, and the decision support system for health care management system. The outcome of the research benefits the knowledge workers, IT staff and other participants to develop the health care management infrastructure of their organizations. The main purposes of building this system are as follows:

- To improve access to information and knowledge at all levels (physicians, hospital administrators and staff, insurer, consumer of health services, pharmacies, and health insurance companies) in order to increase the efficiency and reduce the cost.

- To transform the diverse members (care recipients; providers-physicians, nurses, therapists, diagnosticians; pharmacists; regulators; retail suppliers) of the health care sector into a knowledge network/community of practice.

- To promote self-care by providing easy and friendly access to relevant information, and; enable evidencebased decision-making to improve quality of health care.

The remaining portion of this paper is organized as follows:

Section 2 contains the background material on knowledge management, health care management, benefits of their integration and the research contribution. Section 3 discusses the knowledge management environment with its processes and capabilities. The technical capabilities and infrastructure needed for e-health are discussed followed by the knowledge based decision support architecture, the system interface, and the future research needs. Concluding remarks are provided at the end.

\section{KNOWLEDGE MANAGEMENT}

Knowledge management (KM) is defined as "a justified belief that increases an entity's capacity for effective action" [8]. Knowledge management involves the strategies and processes for identifying, capturing, structuring, sharing and applying an individual's or an organization's knowledge to extract competitive advantage and create sources of sustainable growth [9] [10] [11].

There is clear understanding among the health care managers and practitioners about the transformation of health care industry towards knowledge based industry. All the participants of this community create share and use knowledge to improve the health care service quality and reduce cost. The community includes patients, doctors, paramedical staff, pharmacist, technicians, lab and research staff, administrative staff etc. from different health care organizations. The success of a medical care depends on how effectively and intelligently knowledge is being used to improve the health care process[12] [13].

Management practitioners and theorists have agreed that any process of management of knowledge would involve the 
ability to identify and capture existing knowledge, create new knowledge and transfer existing knowledge throughout the organization [14]. Organizations should try to use KM to manage the flow of information and knowledge as $\mathrm{KM}$ is considered to have utility in the above context as it deals intrinsically with the learning capacity of human resources of a company.

\subsection{Using Health Information Technology}

Health information technology (HIT) [15] may be useful for:

- Minimizing the paperwork by introducing electronic health records for patients.

- Rapid retrieval as well as fast and reliable communication of electronic health records to distant places using modern information and communication technology.

- Decision making based on analysis of patient's history and current data will increase overall efficiency even in remote areas.

- Reduction of health care costs by eliminating the repetition of tests by different doctors.

- Improvement in the quality of care by lessening of medical errors due to inaccurate and untimely information.

\subsection{Privacy and Security Issues}

As ICT use increases further in the health sector, some privacy and security issues will arise [15].

- Over time, all of the health information will be available electronically, not only to the patient and doctor but also to other health care providers and health plan.

- Because many organizations and people may have access to health information there will be concerns about the privacy and security of health information technology tools. Over the past several years there have been security breaches in hospitals and doctors' offices resulting in medical identify theft. Access to personal information may allow thieves to counterfeit medical services bills.

In order to avoid these issues, the doctors, health care providers and health plan must keep the information private by teaching their staff regarding how information may and may not be used and shared. Similarly, appropriate steps must be taken to keep health information secure.

\section{IT IN HEALTHCARE}

\subsection{Health ontology}

Ontology is a specification of conceptualization of knowledge or in other words formal vocabulary of a certain knowledge domain such as web ontology or health ontology. Ontology refers to the definition of objects, events, actions, properties, constraints and relations that exist among different objects in a formal way. Ontologies provide a sound basis for sharing domain knowledge between human and machine and as well as machine to machine. Defining ontology helps to understand the semantic relationship that can be later used for knowledge representation and sharing.

\subsection{Ontologies for Medical Applications}

It is very important to develop ontology for health care and medical application regarding healthcare services or clinical procedures in line with the standard clinical guidelines [16]. The medical ontology includes patients, diseases, symptoms, medicines, lab findings \& reports and their treatment. There must be a generic ontological framework where health related ontology can be built and is integrated with existing libraries. One such example is ONIONS, [17] which integrates the domain ontology using a library of generic ontological theories. Another example is DOLCE [18] which is based on ON9.2 library. For conceptual development an ontology which covers medical and hospital information is required to develop the patient data management (PDM) system [19] [20] [21].

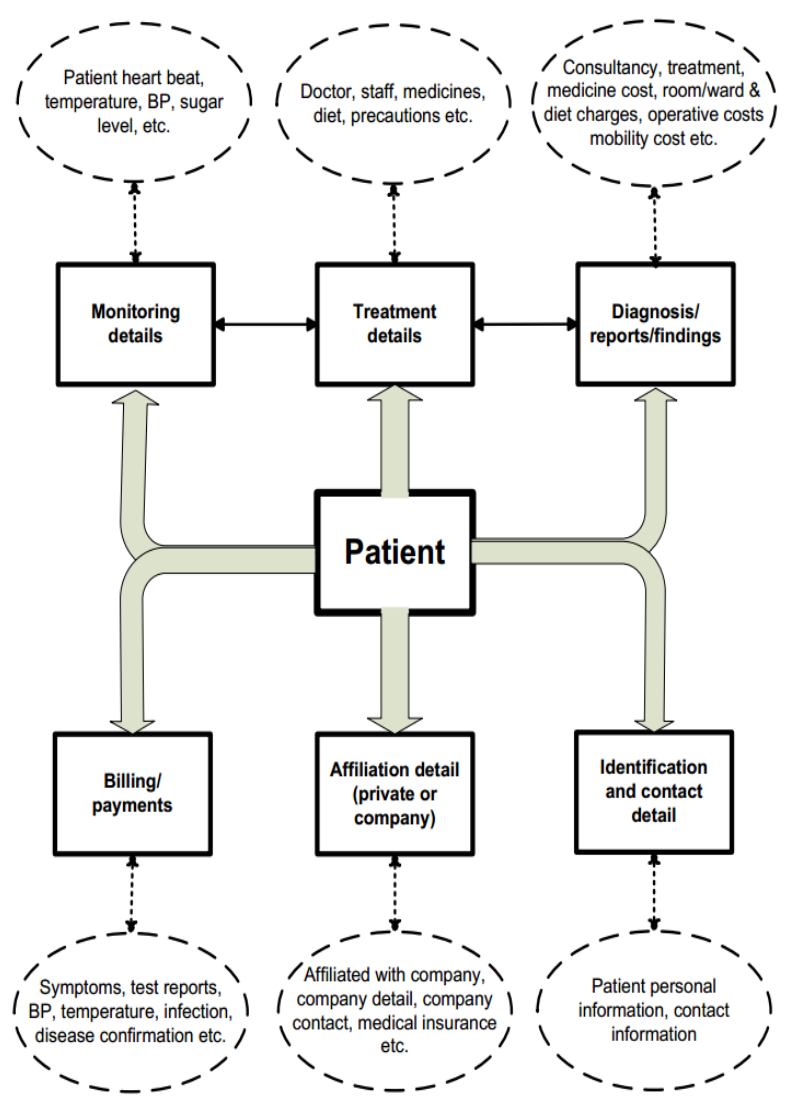

Fig. 1: Electronic Patient Health Record

\subsection{Healthcare information systems}

An information system is a system that provides a means of information storage, processing, and retrieval, so that a user may query and receive the information from the system. An Ontology-based Information System is an information system in which ontology represents a means to access, query, and manipulate the underlying data instances [22]. Ontologybased information system often provides some supports for the semantics.

The main objective of healthcare information system is to provide better service and care to the patients. This includes treatment, monitoring, diagnosis, test results and other services such as patient handling, care, information access and also financial aspect such as billing and information. There are other important entities on HIS which includes doctors, physicians, nurses, staff, lab and OT technicians, and administrators. The traditional patient-doctor relationship has now changed to a healthcare team enabled system involving other entities that facilitate in provision of better care. Information bounds these entities with all other components, in order to provide information sharing. 


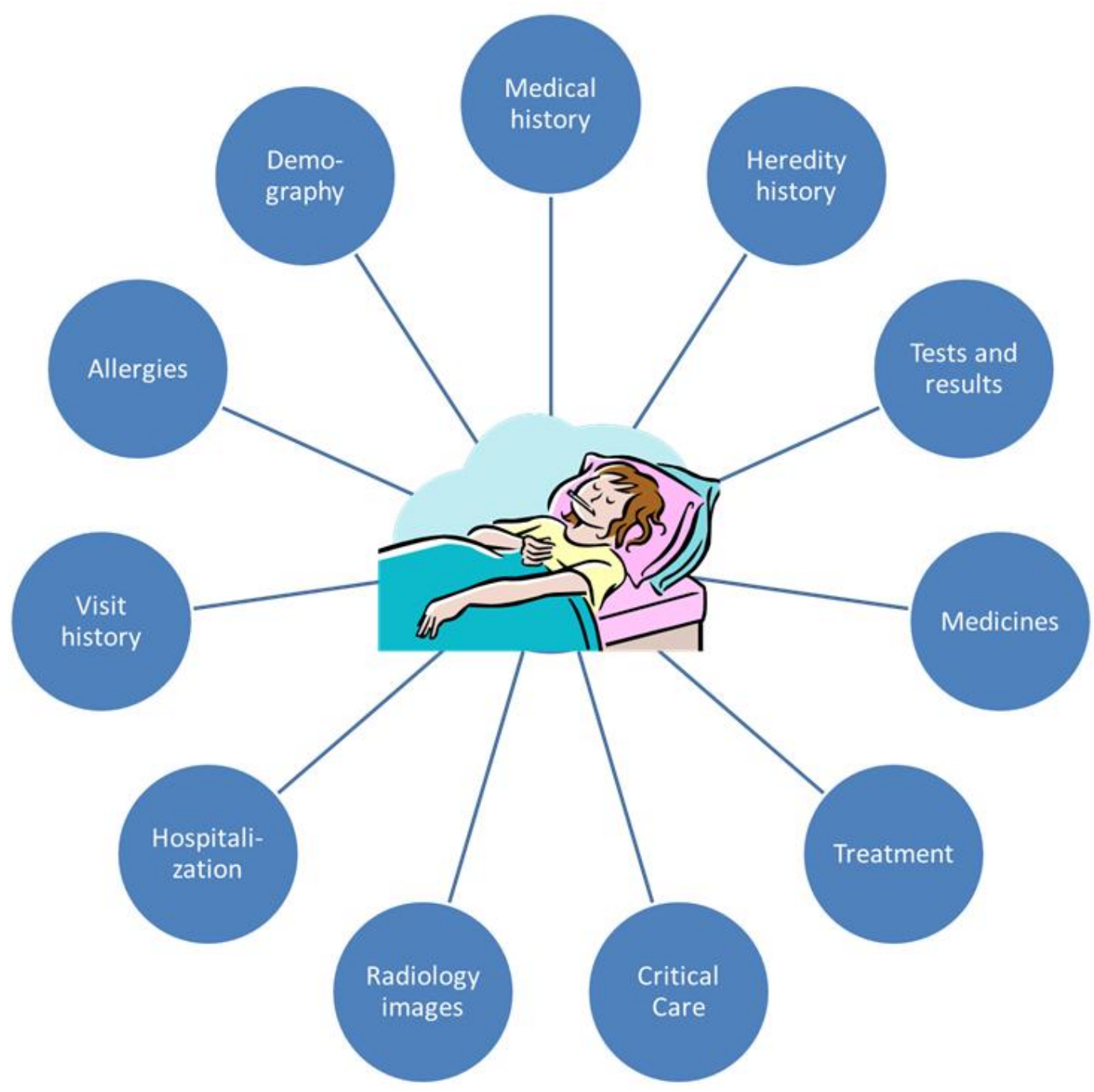

Fig. 2: Conceptual model for Patient Database

This will only become possible if we have the effective up-todate electronic health record system for the patients. A sample Electronic Health Record is shown in Fig. 1. The record stores all the information pertaining to a patient such as company affiliation, monitoring details, treatment and medication, diagnosis and findings, reports, billing information and etc. Another representation of attributes stored in a patient database is provided in Fig. 2.

Knowledge Representation for medical and clinical systems has been subject of research for decades and there are some significant research proposals which are also implemented. This includes the Arden Syntax standard [22], the Guidelines Interchange Format (GLIF) [23], and the Good Electronic Health Record (GEHR) Project [24]. Apart from knowledge representation there are standards for knowledge sharing (exchange) among different institutions, like Health Level 7 (HL7) [25].

Efforts have been made by the researchers to come up with a universal standard for health care and medical information [12] [19] [23]. However there are only very few standards which are being used by health care organizations for sharing of knowledge [24]. One such example is Unified Medical Language System (UMLS), which is used for information integration and retrieval from different health information repositories.

\subsection{Tele medicine}

Telemedicine [26] is a merger of advanced telecom and information technology. It uses the Information and Communication Technologies (ICT) infrastructure to provide healthcare services to organizations and individuals situated at distant locations. It is a relatively new model for interaction with the patients or other important entities such as healthcare service providers, hospitals, pharmacies, physicians and governmental agencies [27]. Moreover, some very advanced technologies such as Tele-surgery have been developed where robotic instruments will perform the surgery on the basis of the audio and visual data received by the surgeon present at a remote or a distant location. This will be useful is situations where immediate transportation of patient to the health facility is not possible [28]. 


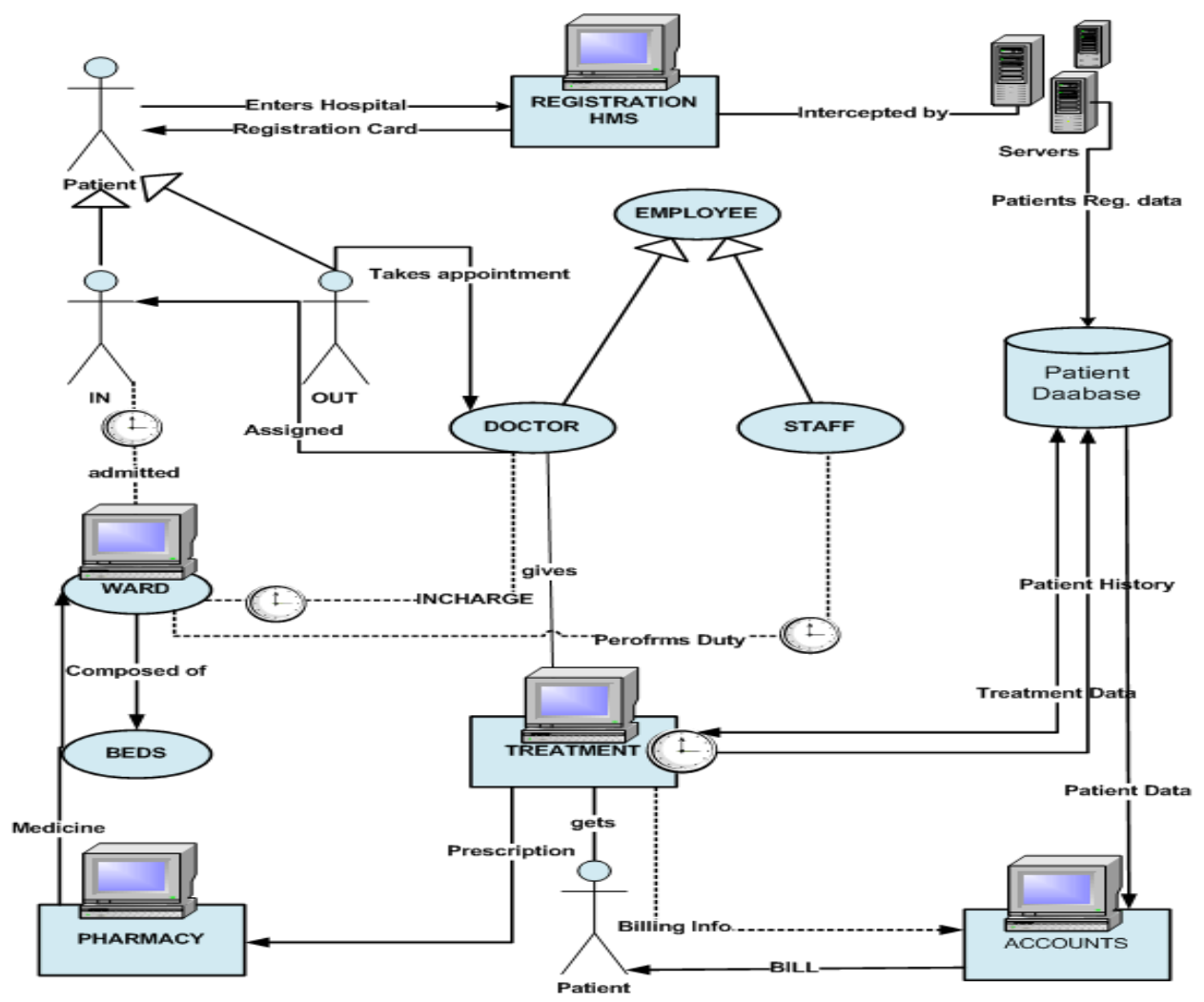

Fig. 3: Healthcare Workflow Model \&ICT

Another important technology is the use of video conferencing, allow clinicians/surgeons and physicians to interact with a distant patients due to time factor in a real time and give his/her expert advice and even interact with the patient. The video conferencing also help the physician to interact with different experts at the same time and make a decision. The modern technology provide the ways to not only store digital images such as MRI, X-Rays and Radiographs but to transmit them effectively and efficiently using teleradiology [29] to the consultants which saves precious time and without any significant loss of data.

\section{KNOWLEDGE MANAGEMENT FRAMEWORK FOR HIS}

\subsection{Healthcare Workflow Management and ICT}

Healthcare workflow model in Fig. 3 represents the complete organization of the system and the flow of data/information among important stakeholders of the system. Patient is considered to be the most important object with in healthcare system because most of the information is generated by patients. This model represents how patient enters into the system, starting with the registration process to admission, diagnosis, treatment and other related activities. It also represents the use of appropriate information and communication technology (ICT), wherever required to improve the healthcare service in terms of timing, quality and functionality.

\subsection{E-health}

ICT plays a vital role in the development of health information system for knowledge management. They assist in the data collection, analysis, management and sharing of information between business processes for health care. The conversion of business processes, communications and transactions in electronic form is also called E-health. There is a fast transformation towards e-health systems by health care organizations due to streamline business processes, reduce costs, and improve overall healthcare services.

\subsection{M-Health}

Advancements in ICT have paved way for provision of costeffective services to the people around the globe. The combination of such wireless technologies with E-health is known as M-health. In general terms, m-health can be defined as mobile computing, medical sensor, and communications technologies for healthcare [30].

The applications include the use of cell phones and other communication devices to gather health data, delivery of healthcare information to doctors, researchers, and patients. It also includes real-time and direct provision of health services. It can help improve clinical outcomes, and contribute to better public health monitoring and education. Fig. 4 highlights the basic concept of the m-Health system. This system will be very handy in locations where there is a dearth of healthcare facilities and infrastructure. The availability of ICT infrastructure will be used to get medical advice from health professionals at remote location through the support centers working round the clock.

Several platforms have been proposed to provide easy to use and cost effective solutions for seamless remote patient doctor interaction over a cellular network [31] [32]. M-Health systems are created as a synergy of emerging mobile medical computing, medical sensor technologies, and communication technologies [30]. 


\subsection{Key requirements for improving Healthcare Services}

In order to improve the effectiveness of healthcare services, following are the requirements that must be taken care of [27].

- Information access to doctors, patients, researchers, healthcare professionals, and others to get desired changes in behaviors and outcomes of all the stakeholders.

- Design less expensive, robust and more realistic methods of testing the effectiveness of alternative clinical practices.

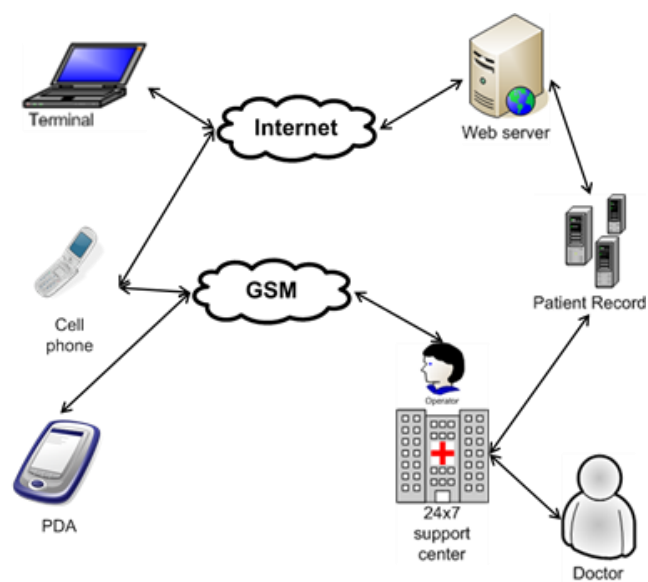

Fig. 4: Concept of M-Health system [27]

- Construct better measures of healthcare outcomes, delivery system characteristics, and other variables that may affect outcomes.

- Development of quantitative and statistical tools that provide vigorous and correct analysis of data.

- Electronic databases for patients must be developed to provide easy and prompt access to their data as well as quick transmission through communication media.

- Decision support systems are required to facilitate the physicians and doctors in decision making.

\subsection{Issues and challenges}

Despite of technological advancements in IT infrastructure health care industry is still facing challenges that need to be resolved [33]. Some of the challenges include

- Complexity of medical data

- absence of industry-wide recognized Electronic Health Care Record (E-HCR) for patients in which medical data about the patient is recorded

- Data entry problems

- Ethical concerns

- Medical knowledge sharing network

- Provisioning of standards

- Security and confidentiality concerns

- Systems integration concerns in clinical practice

- A general lack of awareness of the benefits and risks of IT.
A significant IT challenge in the clinical practice is how to integrate several disparate, standalone, information repositories into a single logical repository-for software applications - to create a single version of truth for all users.

\section{CONCLUSIONS}

Knowledge entails wisdom nevertheless awareness. Health Information System backed by a rich and effective Knowledge Base ensures efficiency in identification, analysis, and selection of optimal action for the patient care. This also improves the access to the patient data and facilitates the decision making process in least amount of time. Sound application of knowledge management can solve half of the problems in healthcare using HIT. Moreover, building the ontology for HIS in m-Health domain is of utmost importance; hence, a conceptual model for health care ontology has been presented in the paper. It is quite obvious that having an efficient and smart semantic database/ knowledgebase, in perusal of tele-medication is essential

A sound knowledge management entails information access among healthcare personals' domain, effective \& efficient data acquisition, swift processing of knowledge for the decision support. A robust ontology in knowledge management shall reduce the challenges and critical issues in HIS that will eventually help us to get full advantage of IT in Healthcare sector

\section{REFERENCES}

[1] Achour, S. L., Dojat, M., Rieux, C., Bierling, P., and Lepage, E. 2001. A UMLS (Unified Medical Language System)based knowledge acquisition tool for rule-based clinical decision support system development. Journal of the American Medical Informatics Association, 8 , pp.351-360.

[2] McDonald, C. J., Overhage, J. M., Dexter, P. R., Blevins, L., Meeks Johnson, J. , Suico, J.G., Tucker, M.C., and Schadow, G. 1998. Canopy computing: using the web in clinical practice. Journal of the American Medical Association, 280 (15), 1325-1329.

[3] Forgionne, G. A., Gangopadhyay, J. A., Klein, R., and Eckhardt, R. 1999a. A decision technology system for health care electronic commerce. Topics in Health Information Management, 20(1), pp. 31-41.

[4] Forgionne, G. A., Gangopadhyay, A., Klein, J. A., and Eckhardt, R. 1999b. Electronic commerce as an enabler of efficient healthcare decision making. Electronic Markets, 9(1), pp. 1-5

[5] Hunt, D. L., Haynes, R. B., Hanna, S. E., and Smith, K. 1998. Effects of computer-based clinical decision support systems on physician performance and patient outcomes: a systematic review. JAMA, 64, pp. 15-37.

[6] Zitner, D., Paterson, G., and Fay, D. 1998. Methods in health decision support systems: Methods for identifying pertinent and superfluous activity. In: Tan J. (Ed.), Health decision support systems. Gaithersburg, MD: Aspen Press.

[7] Sim, I., Gorman, P., Greenes, A., Haynes, R. B., Kaplan, B., Lehmann, H., and Tang,P.C.2001. Clinical decision support systems for the practice of evidence-based medicine. Journal of the American Medical Informatics Association, 8, pp. 527-534. 
[8] Alavi, M., and Leidner, D. E. 2001. Review: Knowledge management and knowledge management systems: conceptual foundations and research issues. MIS Quarterly, 25(1), pp. 107-136.

[9] Davenport, T. H., and Prusak, L. 1998. Working knowledge: How organizations manage what they know. Boston, MA: Harvard Business School.

[10] Holsapple, C. W., and Joshi, K. D. 2002. The evolution of knowledge management frameworks. In S. Barnes (Ed.), Knowledge management systems: Theory and practice. London: International Thomson Press.

[11] Liebowitz, J. 2000.Building organizational intelligence - $A$ knowledge management primer. Boca Raton, FL: CRC Press.

[12] Kohli, R., Tan, J. K., Piontek, F. A., Ziege, D. E., and Groot, H. 1999. Integrating cost information with health management support system: an enhanced methodology to assess health care quality drivers. Topics in Health Information Management, 20(1), pp. 80-95.

[13] Jadad, A. R., Haynes, R. B., Hunt, D., and Browman, G. P. 2000. The Internet and evidence-based decisionmaking: a needed synergy for efficient knowledge management in health care. CMAJ, 162(3), 362-365.

[14] Bolisani, E., and Scarso, E. 1999. Information technology management: a knowledge based perspective, Technovation, 19, pp. 209-217, 1999.

[15] Health-IT-Overview. Retrieved from http://healthinsurance.about.com/od/healthinsurancebasic s/a/health_IT_overview.htm

[16] Mahmood, N., Burney, A., and Ahsan, K. 2012. Generic Temporal and Fuzzy Ontological Framework for Developing Temporal-Fuzzy Database Model for Managing Patient's Data. Journal of Universal Computer Science, 18(2), pp. 177-193.

[17] Gangemi, A., Pisanelli, M. D., and Steve, G. 1999. An overview of the ONIONS project: applying ontologies to the integration of medical terminologies, Data and Knowledge Engineering, 31(2), pp. 183-220.

[18] Masolo, C., Borgo, S., Gangemi, A., Guarino, N., Oltramari, A., Schneider, L.2002. The WonderWeb Library of Foundational Ontologies and the DOLCE Ontology. Wonderweb Deliverable D17. Retrieved from: www.loa-cnr.it, 2002.

[19] Mahmood, N., and Burney, A. 2011. Temporal and Fuzzy Relational Databases, LAP Germany.

[20] Burney, A., Mahmood, N., and Abbas, Z. 2012. Advances in Fuzzy Rough Temporal Databases. In Proc 11th WSEAS International Conference on AIKED, University of Cambridge UK, pp 237-242.
[21] Burney, A., Mahmood, N., and Ahsan, K. 2010. TempRPDM: A Conceptual Temporal Relational Model for Managing, Patient Data. In Proc. Int. WSEAS conference AIKED, University of Cambridge UK, pp. 237-243.

[22] Hripcsak, G. 1994. Writing Arden Syntax Medical Logic Modules, In: Computers in Biology and Medicine, $\mathrm{pp}$ 331-363.

[23] Deibel, R. A. Introduction to the InterMed Common Guideline Model and Guideline Interchange Format (GLIF). Brigham and Women's Hospital. Harvard Medical School. Boston. November, 1996.

[24] The Good Electronic Health Record. Retrieved from: http:// www.gehr.org.

[25] HL7. Health Level 7. Retrieved from: http://www.hl7.org.

[26] Bashshur, R. L., Puskin, D., and Silva, J., eds. Second Invitational Consensus Conference on Telemedicine and the National Information Infrastructure: Augusta, Georgia, May 2-4, 1995. Telemedicine Journal, 1(4), 321-375, 1995.

[27] Burney, A., Mahmood, N., and Abbas, Z. 2010. Information and Communication Technology in Healthcare Management Systems: Prospects for Developing Countries. International Journal of Computer Applications 4(2):27-32, July 2010. Published By Foundation of Computer Science.

[28] Guthart, G.S., and Salisbury, J.K. 2000. The Intuitive Telesurgery System: Overview and Application. In: Proc IEEE ICRA, pp.618 -621.

[29] Gitlin, J. N. 1994. Introduction in understanding Teleradiology. Harrisburg, Pa.: Society for Computer Applications in Radiology.

[30] Istepanian, R. S. H., Jovanov E., and Zhang Y.T. 2004. Guest Editorial Introduction to the Special Section on MHealth: Beyond Seamless Mobility and Global Wireless Health-Care Connectivity. IEEE Transactions on Information Technology in Biomedicine, 8(4), pp. 405414.

[31] Holopainen, A., Galbiati, F., and Takizawa, K. 2008. Health Gateway Mobile phone technology offers easyto-use and cost-effective telemedicine services for rural and remote areas, Journal of eHealth Technology and Application, 5(3), pp. 261-266

[32] Jovanov, E., Milenkovic, A., Otto, C., andGroen P.C. 2005. A wireless body area network of intelligent motion sensors for computer assisted physical rehabilitation, Journal of Neuro Engineering and Rehabilitation, 2(6).

[33] Blumenthal, D.1999. Healthcare format the close of the 20thcentury. New England Journal of Medicine, 340(24), pp. 1916-1920. 